\title{
Evaluation of a random access analyser: BM/Hitachi 911
}

Taweesook Kanluan, Surapon Tangvarasittichai and Orathai Tangvarasittichai

Department of Clinical Chemistry, Faculty of Medical Technology, Mahidol University, Siriraj Hospital, Bangkok 10700, Thailand

The performance of Boehringer Mannheim's BM/Hitachi 911 was evaluated for three months. The mean coefficient of variation $(C V)$ of the within-run and between-run imprecision of the 16 analytes were less than $1 \cdot 16 \%$ (range $0 \cdot 47-2.38 \%$ ) and $1.35 \%$ (range $0.62-2.93 \%$ ), respectively. A linearity study for the various assays covered clinically important levels. No relevant drift was observed during an eight-hour assay nor was any sample-related carry-over detected. In all cases, the regression analyses (slopes) of the results obtained from BM/Hitachi 911 and 717 were between the extreme values of 0.94 and 1.05. During the three months of operation, no major problem was encountered. The BM/Hitachi 911 was found to be easily operated, to require minimal attention and simple daily maintenance during operation.

\section{Introduction}

Objective analytical performance evaluations are important to clinical laboratories when looking at the selection of new instruments [1-4]. The $\mathrm{BM} /$ Hitachi 911 is a very recent selective access analyser from Boehringer Mannheim GmbH. The photometric unit of the 911 allows the grating spectrophotometer unit to be used in monochromatic or bichromatic mode at 12 fixed wavelengths. The cycle time per test is 20 seconds and the throughput is 360 photometric tests an hour; throughput can be increased if the ISE unit is used.
The two-reagent disk units contain all the test materials necessary for 52 different analytical procedures with 800 routine samples, 800 rerun samples and 200 stat samples. The sample volumes range from $3-50 \mu \mathrm{l}$, in $1 \mu \mathrm{l}$ stepwise increments. The reagent probe is capable of delivering maximum reagent volume of $350 \mu \mathrm{l}$ and minimum volume of $250 \mu \mathrm{l}$. The analyser tested was equipped with an optical bar-code reader for a primary tube in sample disks and in both reagent disks and an RS-232 interface allows a bidirectional link to a host computer.

The study reported in this paper evaluated the performance of BM/Hitachi 911 with 16 analytes (see table 1). Within-run and between-run imprecisions, linearity, analyte drift, sample carry-over and correlation are reported.

\section{Materials and methods}

\section{Instruments}

A BM/Hitachi 717 (Boehringer Mannheim GmbH) was used for comparison with the BM/Hitachi 911 (Boehringer Mannheim GmbH)

\section{Materials:}

All reagents and calibrators, unless otherwise stated, were from Boehringer Mannheim $\mathrm{GmbH}$ and were prepared as described in the manufacturer's literature.

Table 1. Test, method and assay condition used on the BM/Hitachi 717 and BM/Hitachi 911.

\begin{tabular}{|c|c|c|c|c|c|}
\hline \multirow[b]{3}{*}{ Analyte } & \multirow[b]{3}{*}{ Method } & \multirow{2}{*}{\multicolumn{2}{|c|}{ Wavelength (nm) }} & \multicolumn{2}{|c|}{ Volume used $(\mu \mathrm{l})$} \\
\hline & & & & \multirow[b]{2}{*}{ Sample } & \multirow{2}{*}{$\begin{array}{l}\text { 'Total } \\
\text { reagent }\end{array}$} \\
\hline & & Main & Sub & & \\
\hline Glucose & Glucose oxidase & 505 & 700 & 3 & 253 \\
\hline BUN & Urease (UV) & 340 & 415 & 4 & 404 \\
\hline Greatinine & Jaffé reaction & 505 & 570 & 15 & 327 \\
\hline Uric acid & Uricase-POP-PAP & 505 & 700 & 7 & 307 \\
\hline Cholesterol & Cholesterol oxidase-PAP & 505 & 700 & 3 & 303 \\
\hline Triglyceride & GPO-PAP & 505 & 700 & 3 & 303 \\
\hline Total protein & Biuret & 546 & 700 & 7 & 507 \\
\hline Albumin & $\mathrm{BCG}$ & 600 & 700 & 3 & 353 \\
\hline Total bilirubin & Dichlorophenyl (DPD) method & 570 & 700 & 7 & 307 \\
\hline AST & SCE method & 340 & 376 & 15 & 290 \\
\hline AL'T & SCE method & 340 & 376 & 15 & 290 \\
\hline Alk. phosphatase & PNP & 415 & 700 & 11 & 311 \\
\hline Total calcium & O-cresolphthalein complexone & 546 & 700 & 10 & 360 \\
\hline Phosphate & Ammonium molybdate (UV) & 340 & 376 & 5 & 365 \\
\hline $\mathrm{LDH}$ & Pyruvate-lactate & 340 & 376 & 7 & 307 \\
\hline CK & Optimized standard method (UV) & 340 & 415 & 7 & 307 \\
\hline
\end{tabular}


Specimens:

One hundred serum samples, ranging from normal to pathological values, were used in the study. Each serum was divided equally and assayed either in the BM/Hitachi 911 or 717. The comparative study was obtained by regression analysis of the values of each serum for 16 analytes determined using minimized sum of squares. The linearity study was carried out using high level concentration specimens.
Control sera:

The control sera used were:

(1) Boehringer Precinorm lot 175303 (Germany).

(2) Corning lot 020002, 020103, 025103 and 037101 (USA). In method calibration, the same calibrator (lot 759350) was used on both of the BM/Hitachi 911 and 717 .

Table 2. Within-run and between-run imprecision of 16 analytes at three concentrations $(\mathcal{N}=20)$.

\begin{tabular}{|c|c|c|c|c|c|c|}
\hline \multirow{2}{*}{$\begin{array}{l}\text { Analyte } \\
\text { (unit) }\end{array}$} & \multicolumn{2}{|c|}{ Within-run } & \multirow{2}{*}{$\begin{array}{c}\text { Imprecision } \\
\mathrm{CV}(\%)\end{array}$} & \multicolumn{2}{|c|}{ Between-run } & \multirow{2}{*}{$\begin{array}{c}\text { Imprecision } \\
\mathrm{CV}(\%)\end{array}$} \\
\hline & Mean & $\mathrm{SD}$ & & Mean & $\mathrm{SD}$ & \\
\hline \multirow{3}{*}{$\begin{array}{l}\text { Glucose } \\
(\mathrm{mmol} / \mathrm{l})\end{array}$} & $6 \cdot 52$ & 0.05 & $0 \cdot 63$ & $6 \cdot 54$ & 0.04 & $0 \cdot 68^{a}$ \\
\hline & $9 \cdot 72$ & $0 \cdot 11$ & 1.09 & $9 \cdot 74$ & $0 \cdot 11$ & $1 \cdot 12^{b}$ \\
\hline & $17 \cdot 00$ & $0 \cdot 11$ & $0 \cdot 60$ & $17 \cdot 05$ & $0 \cdot 11$ & $0 \cdot 62^{c}$ \\
\hline \multirow{3}{*}{$\begin{array}{l}\text { BUN } \\
(\mathrm{mmol} / \mathrm{l})\end{array}$} & $4 \cdot 13$ & $0 \cdot 04$ & $0 \cdot 93$ & $4 \cdot 18$ & 0.05 & $1 \cdot 23^{a}$ \\
\hline & $8 \cdot 52$ & $0 \cdot 15$ & $1 \cdot 78$ & $8 \cdot 62$ & $0 \cdot 17$ & $1 \cdot 95^{b}$ \\
\hline & $13 \cdot 04$ & $0 \cdot 18$ & $1 \cdot 42$ & $13 \cdot 13$ & $0 \cdot 20$ & $1 \cdot 56^{c}$ \\
\hline \multirow{3}{*}{$\begin{array}{l}\text { Creatinine } \\
(\mu \mathrm{mol} / \mathrm{l})\end{array}$} & $189 \cdot 17$ & $4 \cdot 51$ & $2 \cdot 38$ & $190 \cdot 94$ & $5 \cdot 39$ & $2 \cdot 82^{a}$ \\
\hline & $369 \cdot 07$ & $7 \cdot 60$ & $2 \cdot 06$ & $374 \cdot 90$ & $8 \cdot 31$ & $2 \cdot 22^{b}$ \\
\hline & $720 \cdot 46$ & $6 \cdot 36$ & $0 \cdot 88$ & $721 \cdot 32$ & $7 \cdot 16$ & $0 \cdot 99^{c}$ \\
\hline \multirow{3}{*}{$\begin{array}{l}\text { Uric acid } \\
(\mu \mathrm{mol} / \mathrm{l})\end{array}$} & $294 \cdot 00$ & $3 \cdot 03$ & 1.03 & $296 \cdot 79$ & $4 \cdot 88$ & $1 \cdot 64^{a}$ \\
\hline & 513.99 & $4 \cdot 82$ & $0 \cdot 94$ & $590 \cdot 73$ & $5 \cdot 35$ & $0 \cdot 91^{b}$ \\
\hline & $614 \cdot 83$ & $5 \cdot 53$ & $0 \cdot 90$ & $622 \cdot 26$ & $5 \cdot 71$ & $0.92^{c}$ \\
\hline \multirow{3}{*}{$\begin{array}{l}\text { Cholesterol } \\
(\mathrm{mmol} / \mathrm{l})\end{array}$} & $3 \cdot 10$ & $0 \cdot 02$ & $0 \cdot 68$ & $3 \cdot 11$ & 0.03 & $0 \cdot 84^{a}$ \\
\hline & $3 \cdot 36$ & 0.59 & 1.75 & $3 \cdot 36$ & 0.06 & $1 \cdot 82^{b}$ \\
\hline & $4 \cdot 44$ & $0 \cdot 02$ & 0.47 & $4 \cdot 45$ & 0.02 & $0 \cdot 52^{c}$ \\
\hline \multirow{3}{*}{$\begin{array}{l}\text { Triglyceride } \\
(\mathrm{mmol} / \mathrm{l})\end{array}$} & $1 \cdot 32$ & $0 \cdot 01$ & $0 \cdot 76$ & $1 \cdot 32$ & $0 \cdot 01$ & $0 \cdot 76^{a}$ \\
\hline & $1 \cdot 60$ & $0 \cdot 01$ & $0 \cdot 63$ & $1 \cdot 61$ & 0.01 & $0 \cdot 62^{b}$ \\
\hline & $1 \cdot 73$ & $0 \cdot 02$ & $1 \cdot 15$ & 1.74 & 0.02 & $1 \cdot 15^{c}$ \\
\hline \multirow{3}{*}{$\begin{array}{l}\text { Total protein } \\
(\mathrm{g} / \mathrm{l})\end{array}$} & $50 \cdot 81$ & 0.98 & 1.93 & $51 \cdot 01$ & 1.01 & $1.98^{a}$ \\
\hline & $51 \cdot 00$ & 0.31 & $0 \cdot 61$ & $51 \cdot 50$ & $0 \cdot 40$ & $0.77^{b}$ \\
\hline & $61 \cdot 13$ & 0.35 & 0.57 & $62 \cdot 00$ & $0 \cdot 41$ & $0 \cdot 66^{c}$ \\
\hline \multirow{3}{*}{$\begin{array}{l}\text { Albumin } \\
(\mathrm{g} / \mathrm{l})\end{array}$} & $30 \cdot 20$ & $0 \cdot 30$ & $0 \cdot 99$ & $31 \cdot 00$ & $0 \cdot 45$ & $1 \cdot 45^{a}$ \\
\hline & $36 \cdot 10$ & 0.51 & $1 \cdot 41$ & $36 \cdot 40$ & 0.56 & $1 \cdot 54^{b}$ \\
\hline & $36 \cdot 53$ & 0.52 & $1 \cdot 42$ & $36 \cdot 78$ & 0.64 & $1 \cdot 74^{c}$ \\
\hline \multirow{3}{*}{$\begin{array}{l}\text { Total bilirubin } \\
(\mu \mathrm{mol} / \mathrm{l})\end{array}$} & 35.91 & 0.72 & $2 \cdot 00$ & $37 \cdot 62$ & $0 \cdot 87$ & $2 \cdot 32^{a}$ \\
\hline & $72 \cdot 73$ & $1 \cdot 42$ & 1.95 & $73 \cdot 89$ & 1.56 & $2 \cdot 10^{b}$ \\
\hline & $119 \cdot 87$ & 1.44 & $1 \cdot 20$ & $121 \cdot 41$ & 1.47 & $1 \cdot 21^{c}$ \\
\hline $\mathrm{AST}$ & $50 \cdot 60$ & $0 \cdot 49$ & 0.96 & $51 \cdot 20$ & 0.91 & $1 \cdot 78^{a}$ \\
\hline \multirow{2}{*}{$(\mathrm{U} / \mathrm{l})$} & $104 \cdot 92$ & 1.89 & $1 \cdot 80$ & $108 \cdot 12$ & 1.98 & $1 \cdot 83^{b}$ \\
\hline & $205 \cdot 40$ & 1.92 & 0.93 & $206 \cdot 10$ & 1.94 & $0 \cdot 94^{c}$ \\
\hline AL'T & $44 \cdot 60$ & $0 \cdot 54$ & $1 \cdot 21$ & $45 \cdot 10$ & 0.62 & $1 \cdot 37^{a}$ \\
\hline \multirow[t]{2}{*}{$(\mathrm{U} / \mathrm{l})$} & $88 \cdot 85$ & $1 \cdot 14$ & $1 \cdot 28$ & $89 \cdot 94$ & 1.25 & $1 \cdot 38^{b}$ \\
\hline & $106 \cdot 80$ & 0.94 & 0.88 & $107 \cdot 20$ & 1.21 & $1 \cdot 13^{c}$ \\
\hline ALP & $74 \cdot 31$ & 1.03 & $1 \cdot 38$ & $75 \cdot 24$ & $1 \cdot 13$ & $1 \cdot 49^{a}$ \\
\hline \multirow[t]{2}{*}{$(\mathrm{U} / \mathrm{l})$} & $224 \cdot 81$ & $2 \cdot 14$ & 0.95 & $225 \cdot 40$ & $2 \cdot 41$ & $1 \cdot 07^{b}$ \\
\hline & $317 \cdot 46$ & $4 \cdot 07$ & $1 \cdot 28$ & $318 \cdot 42$ & $4 \cdot 10$ & $1 \cdot 29^{c}$ \\
\hline \multirow{3}{*}{$\begin{array}{l}\text { Total calcium } \\
(\mathrm{mmol} / \mathrm{l})\end{array}$} & $2 \cdot 13$ & $0 \cdot 05$ & $2 \cdot 35$ & $2 \cdot 14$ & 0.06 & $2 \cdot 93^{a}$ \\
\hline & $3 \cdot 07$ & $0 \cdot 05$ & 1.70 & $3 \cdot 12$ & 0.07 & $2 \cdot 40^{b}$ \\
\hline & $3 \cdot 36$ & $0 \cdot 05$ & $1 \cdot 48$ & $3 \cdot 38$ & $0 \cdot 07$ & $2 \cdot 07^{c}$ \\
\hline Phosphate & 1.59 & $0 \cdot 02$ & $1 \cdot 14$ & $1 \cdot 61$ & 0.02 & $1 \cdot 22^{a}$ \\
\hline \multirow[t]{2}{*}{$(\mathrm{mmol} / \mathrm{l})$} & $2 \cdot 38$ & 0.03 & $1 \cdot 10$ & $2 \cdot 41$ & 0.03 & $1 \cdot 31^{b}$ \\
\hline & $2 \cdot 41$ & 0.03 & 1.05 & $2 \cdot 42$ & 0.03 & $1 \cdot 25^{c}$ \\
\hline $\mathrm{LDH}$ & $292 \cdot 10$ & $2 \cdot 16$ & $0 \cdot 74$ & $292 \cdot 50$ & $2 \cdot 46$ & $0 \cdot 84^{a}$ \\
\hline \multirow[t]{2}{*}{$(\mathrm{U} / \mathrm{l})$} & $586 \cdot 69$ & $4 \cdot 10$ & $0 \cdot 70$ & $589 \cdot 97$ & 4.90 & $0 \cdot 83^{b}$ \\
\hline & $955 \cdot 20$ & $5 \cdot 10$ & 0.53 & $957 \cdot 10$ & $8 \cdot 10$ & $0 \cdot 85^{c}$ \\
\hline CK & $248 \cdot 10$ & $2 \cdot 13$ & $0 \cdot 86$ & $249 \cdot 40$ & $2 \cdot 48$ & $0 \cdot 99^{a}$ \\
\hline \multirow[t]{2}{*}{$(\mathrm{U} / \mathrm{l})$} & $439 \cdot 00$ & $3 \cdot 10$ & $0 \cdot 71$ & $441 \cdot 24$ & $3 \cdot 41$ & $0 \cdot 77^{b}$ \\
\hline & $505 \cdot 00$ & $2 \cdot 71$ & $0 \cdot 54$ & $506 \cdot 20$ & $3 \cdot 41$ & $0 \cdot 67^{c}$ \\
\hline
\end{tabular}

Where: $a$ = Boehringer precinorm lot 175303, Germany; $b$ = Ciba Corning: lot 020002, USA; $c$ = Ciba Corning: lot 037101, USA. 
Table 3. Sample-related carry-over of 16 analytes.

\begin{tabular}{|c|c|c|c|c|c|}
\hline \multirow[b]{2}{*}{ Analyte } & \multirow[b]{2}{*}{ Unit } & \multicolumn{2}{|c|}{ Concentration } & \multirow{2}{*}{$\begin{array}{l}\% \text { Carry-over, when } \\
\text { high as contaminant }\end{array}$} & \multirow{2}{*}{$\begin{array}{l}\% \text { Carry-over, when } \\
\text { low as contaminant }\end{array}$} \\
\hline & & High $(\mathrm{h})$ & Low (1) & & \\
\hline Glucose & $\mathrm{mmol} / \mathrm{l}$ & $16 \cdot 2$ & $4 \cdot 6$ & 0 & $-0 \cdot 34$ \\
\hline BUN & $\mathrm{mmol} / \mathrm{l}$ & $8 \cdot 9$ & $2 \cdot 3$ & $0 \cdot 71$ & -0.57 \\
\hline Creatinine & $\mu \mathrm{mol} / \mathrm{l}$ & 725 & 183 & 0 & $-1 \cdot 22$ \\
\hline Uric acid & $\mu \mathrm{mol} / 1$ & 501 & 291 & 0 & 0 \\
\hline Cholesterol & $\mathrm{mmol} / \mathrm{l}$ & $4 \cdot 0$ & $3 \cdot 2$ & 0 & $-0 \cdot 81$ \\
\hline 'Triglyceride & $\mathrm{mmol} / \mathrm{l}$ & $1 \cdot 8$ & $0 \cdot 9$ & $-1 \cdot 2$ & $-0 \cdot 61$ \\
\hline Total protein & $\mathrm{g} / \mathrm{l}$ & 61 & 48 & 0 & 0 \\
\hline Albumin & $\mathrm{g} / \mathrm{l}$ & 40 & 33 & 0 & 0 \\
\hline Total bilirubin & $\mu \mathrm{mol} / 1$ & 163 & 10 & 0 & $1 \cdot 05$ \\
\hline $\mathrm{AST}$ & $\mathrm{U} / \mathrm{l}$ & 185 & 32 & 0 & -1.07 \\
\hline ALT & $\mathrm{U} / \mathrm{l}$ & 92 & 32 & 0 & $1 \cdot 08$ \\
\hline Alk. phosphatase & $\mathrm{U} / \mathrm{l}$ & 287 & 99 & 0 & $-1 \cdot 36$ \\
\hline 'Total calcium & $\mathrm{mmol} / \mathrm{l}$ & $3 \cdot 1$ & $2 \cdot 3$ & $-1 \cdot 0$ & 0 \\
\hline Phosphate & $\mathrm{mmol} / \mathrm{l}$ & $2 \cdot 3$ & $0 \cdot 9$ & 0 & -1.39 \\
\hline $\mathrm{LDH}$ & $\mathrm{U} / \mathrm{l}$ & 950 & 316 & 0 & $0 \cdot 21$ \\
\hline CK & $\mathrm{U} / \mathrm{l}$ & 515 & 142 & 0 & -1.74 \\
\hline
\end{tabular}

(3) The reagents for determinations of glucose, AST and $\Lambda \mathrm{LT}$ were from Wako (Japan) and Ames (Italy), respectively.

\section{Methods}

The methods and assay conditions used in this study on $\mathrm{BM} /$ Hitachi 717 or 911 are summarized in table 1 .

\section{Results}

\section{Imprecision}

Within-run and between-run imprecision was investigated with three different levels of control sera. The within-run imprecision was assayed 20 times in the same batch, and between-run imprecision was tested using the same sera on 20 consecutive batches. Data on the within-run and between-run imprecision are presented in table 2. The percentage of coefficient of variation $(\% \mathrm{CV})$ of both assays was less than $3 \%$.

\section{Accuracy and linearity}

$\Lambda$ linearity study was performed using a high concentration control serum diluted with isotonic saline. The diluted sera were assayed in duplicate and the mean values obtained. The difference between calculated target and observed value was used for assessing accuracy. The upper limit of each analyte obtained from the study is shown in figure 1. The upper limits were in close agreement with the expected ranges claimed by the manufacturer.

\section{Drift}

The drift of 16 analytes was assayed using two control sera analysed at hourly intervals for eight hours. The value determined at zero hours were performed in triplicate and the subsequent determinations were performed once. The pooled sera were aliquoted in tightly closed vials and kept in a refrigerator. Prior to the assay, the aliquot was transferred to a sample cup and left at room temperature for $10 \mathrm{~min}$. None of the analytes showed a deviation more than $5 \%$ (see figure $2(a)$ and $2(b)$ ).

\section{Sample carry-over}

Carry-over caused by a sample probe was assayed using Bennet's model (6). The assay was performed in three successive sample portions: high concentration $\left(h_{1} \ldots h_{3}\right)$, low concentration $\left(l_{1} \ldots l_{3}\right)$ and same high concentration $\left(h_{1} \ldots h_{3}\right)$. All samples were assayed in triplicate and the percentage carry-over was calculated as follow:

or

$$
\text { High samples }=\frac{h_{1}-h_{2}}{h_{2}} \times 100
$$

$$
\text { Low samples }=\frac{l_{1}-l_{2}}{l_{2}} \times 100
$$

Data presented in table 3 show that there was no appreciable carry-over in any analytes. The overall percentage carry-over was less than $2 \%$.

\section{Correlation}

One hundred samples from normal to pathological levels were divided in half and assayed simultaneously in the $\mathrm{BM} /$ Hitachi 911 or 717 . Table 4 presents the regression analysis of 16 analytes. The extreme slope values obtained were 0.91 and 1.09 , and those for intercepts were -8.22 and $0 \cdot 61$, respectively. This finding suggested that the two instruments performed similarly.

\section{Discussion}

Total analytical imprecision is the summation of the variances arising from both chemical and instrumental factors $[1,7]$. In this study, the CVs of between-run imprecision in three control sera were acceptable $(<3 \%)$. 
T. Kanluan et al. Evaluation of a random access analyser: BM/Hitachi 911
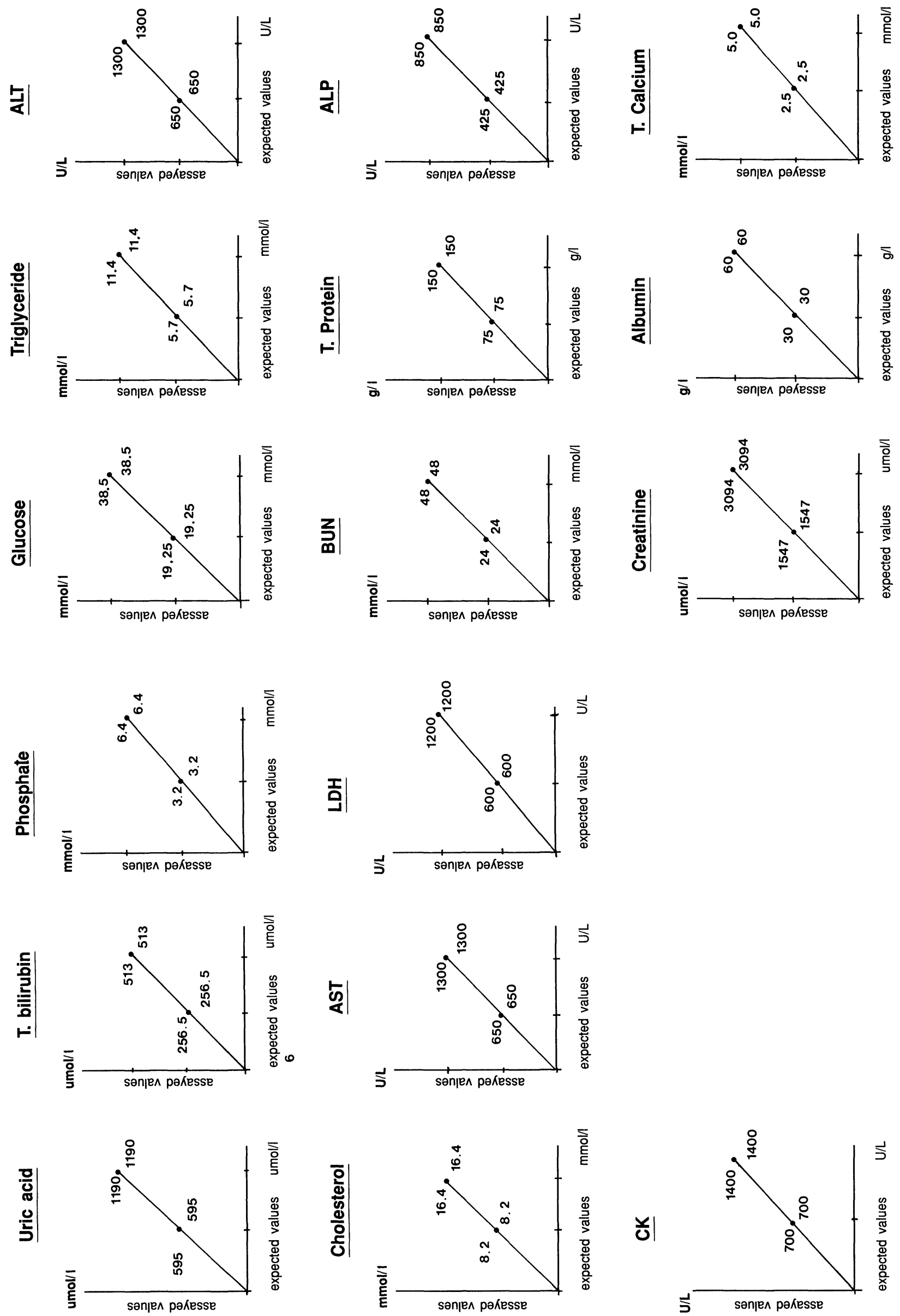

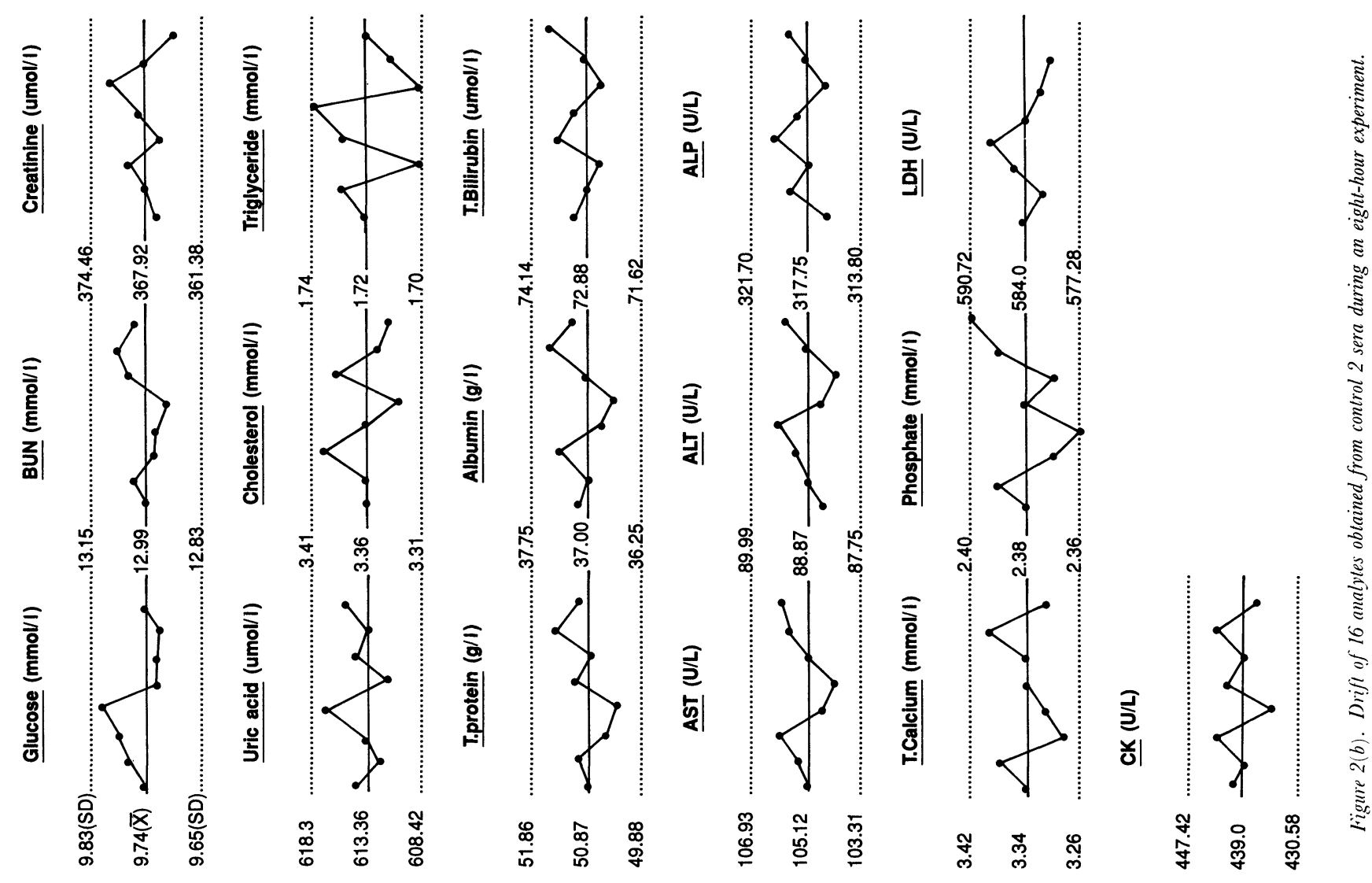

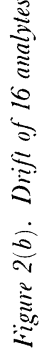
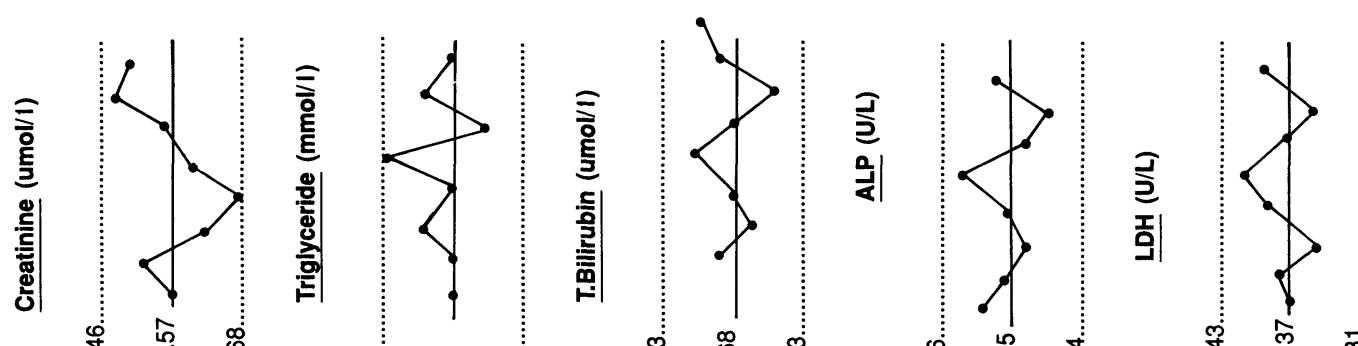

守

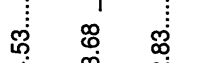

辛
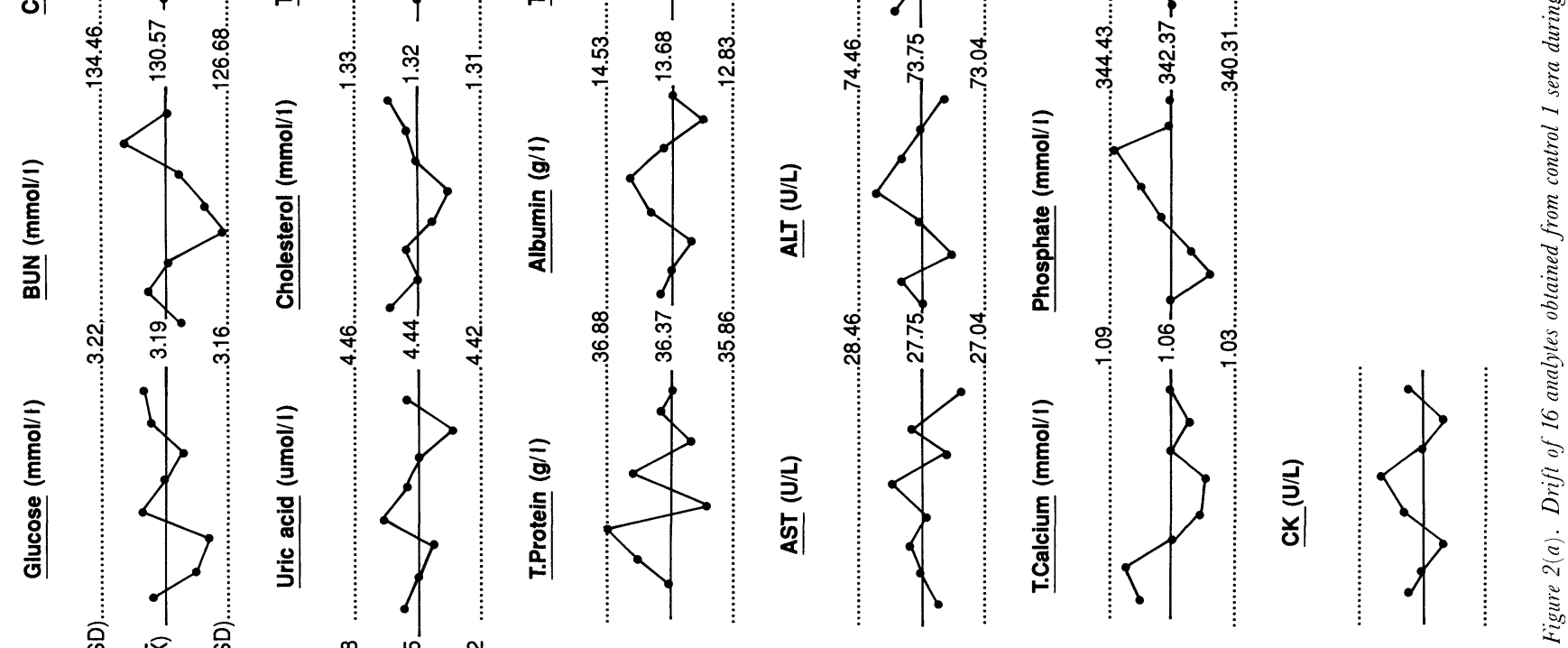

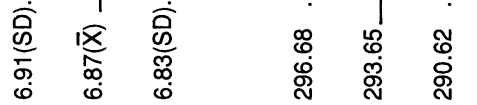

兽

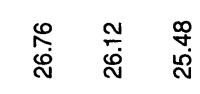

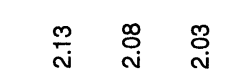

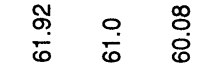


Table 4. Regression analysis of 16 analytes on BM/Hitachi $717(x)$ and $911(y)$, where $\mathcal{N}=100$.

\begin{tabular}{|c|c|c|c|c|}
\hline \multirow[b]{2}{*}{ Analyte } & \multirow[b]{2}{*}{ Unit } & \multicolumn{3}{|c|}{$\begin{array}{l}\text { Regression analysis } \\
\quad(\mathbf{Y}=b x+a)\end{array}$} \\
\hline & & $b$ & $r$ & $a$ \\
\hline Glucose & $\mathrm{mmol} / \mathrm{l}$ & 0.96 & 0.99 & -0.007 \\
\hline BUN & $\mathrm{mmol} / \mathrm{l}$ & $1 \cdot 05$ & 0.98 & -0.023 \\
\hline Greatinine & $\mu \mathrm{mol} / 1$ & $1 \cdot 09$ & 0.99 & $-8 \cdot 22$ \\
\hline Uric acid & $\mu \mathrm{mol} / 1$ & $0 \cdot 99$ & 0.99 & $-7 \cdot 77$ \\
\hline Cholesterol & $\mathrm{mmol} / \mathrm{l}$ & $0 \cdot 94$ & 0.98 & $0 \cdot 003$ \\
\hline Triglyceride & $\mathrm{mmol} / \mathrm{l}$ & 0.97 & 0.99 & $0 \cdot 0002$ \\
\hline 'Total protein & $\mathrm{g} / 1$ & $1 \cdot 01$ & 0.99 & $0 \cdot 47$ \\
\hline Albumin & $\mathrm{g} / 1$ & $0 \cdot 99$ & 0.99 & $0 \cdot 10$ \\
\hline Total bilirubin & $\mu \mathrm{mol} / 1$ & $0 \cdot 91$ & 0.99 & -0.11 \\
\hline AS'T & $\mathrm{U} / \mathrm{l}$ & $1 \cdot 05$ & 0.99 & $0 \cdot 19$ \\
\hline ALT & $\mathrm{U} / \mathrm{l}$ & $1 \cdot 01$ & 0.99 & $-0 \cdot 20$ \\
\hline Alk. phosphatase & $\mathrm{U} / \mathrm{l}$ & $0 \cdot 98$ & 0.99 & $-2 \cdot 83$ \\
\hline Total calcium & $\mathrm{mmol} / \mathrm{l}$ & $0 \cdot 97$ & 0.98 & $0 \cdot 030$ \\
\hline Phosphate & $\mathrm{mmol} / \mathrm{l}$ & $0 \cdot 96$ & 0.99 & -0.006 \\
\hline $\mathrm{LDH}$ & $\mathrm{U} / \mathrm{l}$ & $1 \cdot 01$ & 0.99 & $0 \cdot 61$ \\
\hline CK & $\mathrm{U} / 1$ & 0.99 & 0.99 & $0 \cdot 48$ \\
\hline
\end{tabular}

According to the quality specification for between-run analytical imprecision proposed by a Working Group of EGE-Lab [8], it was shown that the analytical system achieved these specifications in almost all cases (table 5). This finding reflected the good quality spectrophotometer and pipetting systems. However, the mean values for each analyte in the investigation of imprecision were consistently slightly higher between run compared with within run. This could be due to a slight change in the biological matrix during the storage of control serum. Photometric linearity was adequate in all tests (see figure 1) with no drift detected in any various analytes during an eight-hour assay. There is good correlation $(r=0.97-0.99)$ between the results obtained from $\mathrm{BM} /$ Hitachi 911 and 717 . There were no problems during the installation of $\mathrm{BM} / \mathrm{Hitachi}$ 911; and there were no instrument failures during the evaluation study. Laboratory staff learnt to operate and maintain the equipment within three days. The operator's manual and guidelines for trouble-shooting are easily understood.

In conclusion, the $\mathrm{BM} / \mathrm{Hitachi} 911$ fulfilled the acceptance criteria for analytical performance. This instrument is a flexible, convenient and easy-to-use analyser for either batch or random access work. Its design and operational simplicity provides reliable analytical data. The BM/ Hitachi 911 is well-suited to routine operation and emergency analyses for small and medium-sized laboratories, and as a back-up system for large laboratories.
Table 5. Comparison of between-run imprecision proposed by the Working Group of EGE-Lab and BM/Hitachi 911.

\begin{tabular}{|c|c|c|c|}
\hline \multirow[b]{2}{*}{ Analyte } & \multirow{2}{*}{$\begin{array}{l}\text { Working } \\
\text { Group* }\end{array}$} & \multicolumn{2}{|c|}{ BH/Hitachi 911} \\
\hline & & $\% \mathrm{CV}$ & (Mean) \\
\hline Glucose & $2 \cdot 2$ & $1 \cdot 12$ & $(9.74 \mathrm{mmol} / \mathrm{l})$ \\
\hline BUN & $6 \cdot 3$ & 1.95 & $(8.62 \mathrm{mmol} / \mathrm{l})$ \\
\hline Creatinine & $2 \cdot 2$ & $2 \cdot 82$ & $(190.9 \mu \mathrm{mol} / \mathrm{l})$ \\
\hline Uric acid & $4 \cdot 2$ & $1 \cdot 64$ & $(296 \cdot 8 \mu \mathrm{mol} / \mathrm{l})$ \\
\hline Cholesterol & $2 \cdot 7$ & $1 \cdot 82$ & $(3.36 \mathrm{mmol} / \mathrm{l})$ \\
\hline Triglyceride & $11 \cdot 5$ & $1 \cdot 21$ & $(1.74 \mathrm{mmol} / \mathrm{l})$ \\
\hline Total protein & $1 \cdot 4$ & 1.98 & $(51 \cdot 0 \mathrm{~g} / \mathrm{l})$ \\
\hline Albumin & $1 \cdot 4[1 \cdot 8]$ & 1.74 & $(36 \cdot 8 \mathrm{~g} / \mathrm{l})$ \\
\hline Total bilirubin & $11 \cdot 3$ & $2 \cdot 32$ & $(37 \cdot 6 \mu \mathrm{mol} / \mathrm{l})$ \\
\hline AST & $7 \cdot 2$ & $1 \cdot 83$ & $(108 \cdot 1 \mathrm{U} / \mathrm{l})$ \\
\hline ALT & $13 \cdot 6$ & $1 \cdot 38$ & $(89 \cdot 9 \mathrm{U} / \mathrm{l})$ \\
\hline Total calcium & $0 \cdot 9[1.5]$ & $2 \cdot 93$ & $(2 \cdot 14 \mathrm{mmol} / \mathrm{l})$ \\
\hline Phosphate & $4 \cdot 0$ & $1 \cdot 31$ & $(2 \cdot 41 \mathrm{mmol} / \mathrm{l})$ \\
\hline LDH & $3 \cdot 9$ & $0 \cdot 85$ & $(957 \cdot 1 \mathrm{U} / \mathrm{l})$ \\
\hline CK & $20 \cdot 7$ & $0 \cdot 99$ & $(249 \cdot 4 \mathrm{U} / \mathrm{l})$ \\
\hline
\end{tabular}

Where * Proposed quality specification of between-run imprecision $(\%$ CV) by a Working Group of EGE-Lab [8].

[ ] interim quality specifications proposed by the Working Group.

\section{Acknowledgements}

The authors wish to express their sincere thanks to Dr Verawan Veinravi for her constructive criticism and reading the paper and to Nida Dakrabutr for her skillful preparation of this manuscript.

\section{References}

1. Broughton, P. M. G., Gowenlock, A. H., MaCormack, J J. and Neill, D. W., Annals of Clinical Biochemistry, 11 (1974), 207.

2. Fraser, C. G. and Singer, R., Clinical Chemistry, 31 (1985), 667.

3. Kanluan, T., Intaramanee, S. and Tangvorasirtichai, S. et al., Journal of Automatic Chemistry, 13 (1991), 97.

4. Bonini, P., Coriotti, F. and Frangini, C., Journal of Automatic Chemistry, 10 (1988), 167.

5. Okuda, K., Journal of Clinical Chemistry and Clinical Biochemistry, 18 (1980), 947.

6. Bennet, A., Gartelmann, D., Mason, J. I., and Owen, J. A., Clinica Chimica Acta, 29 (1970), 161.

7. Buttner, J., Borth, R., Boutwell, J. H., Broughton, P. M. G. and Bowyer, R. C., Journal of Clinical Chemistry and Clinical Biochemistry, 18 (1978), 69.

8. Fraser, G. G., Hyltoft, Peterson, P., Ricos, C. and Hacokel, R., Journal of Clinical Chemistry and Clinical Biochemistry, 30 (1992) 311. 


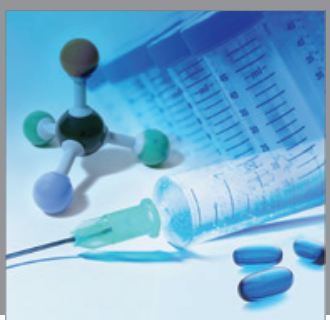

International Journal of

Medicinal Chemistry

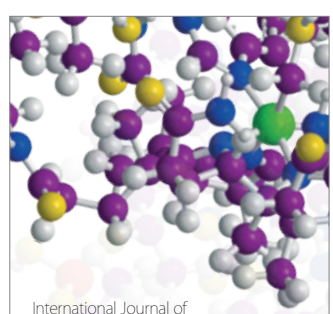

Carbohydrate Chemistry

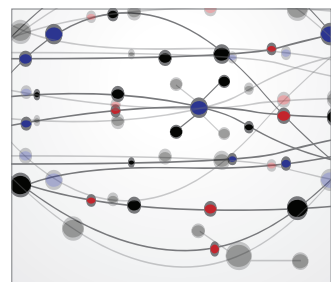

The Scientific World Journal
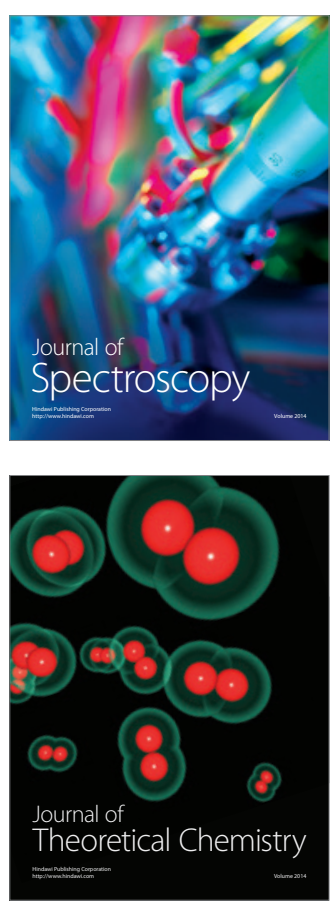
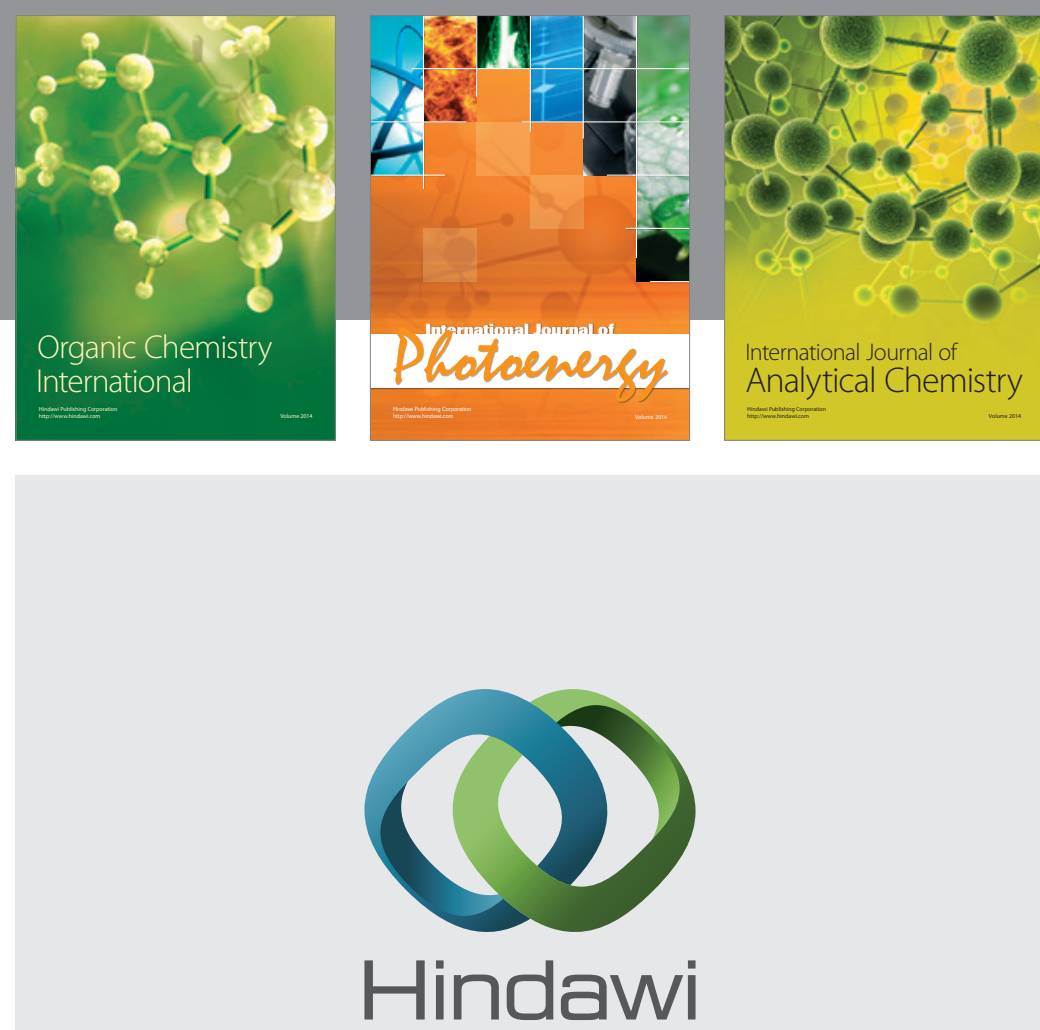

Submit your manuscripts at

http://www.hindawi.com
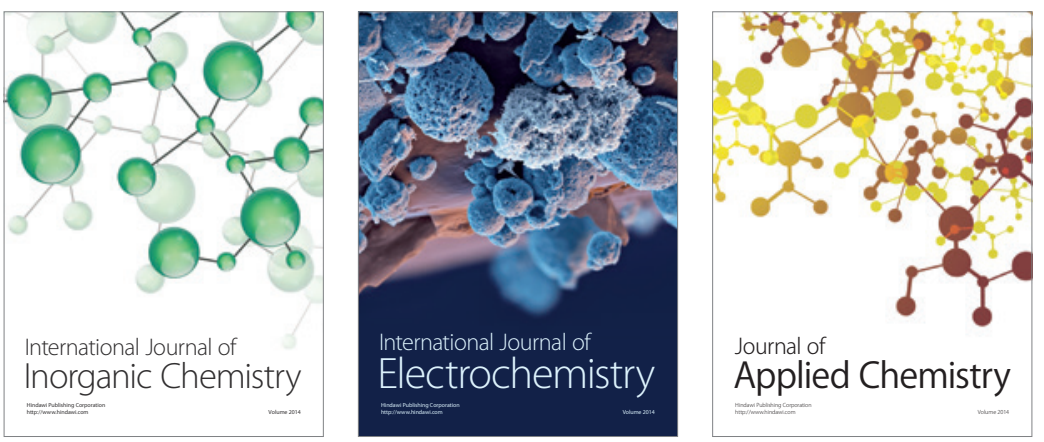

Journal of

Applied Chemistry
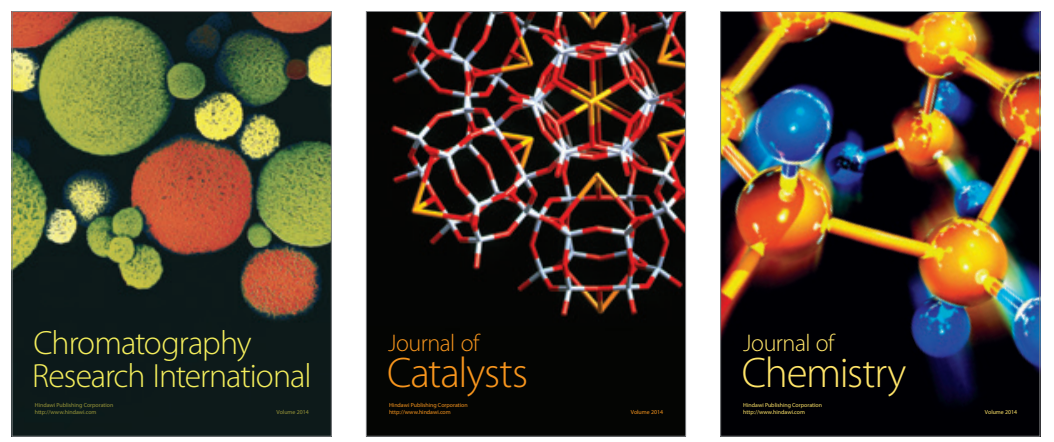
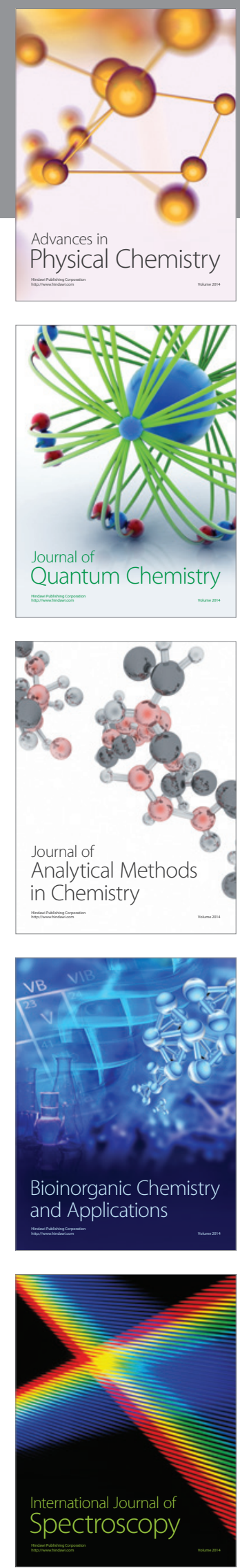